\title{
AIR-WATER COOLING IN THE BUBBLE COLUMN
}

\author{
Gennadiy Medvedev, Evgeniy Cherepanov \\ National Research Tomsk Polytechnic University, 634050 Tomsk, Russia
}

\begin{abstract}
The processes of air-water interaction are in general use in engineering. Air and water tend to be the most popular heat-storage media. In particular, the process of airwater cooling is widely used in water cooling at thermal electric power stations in cooling towers, in systems of in-plant water recirculation, etc.
\end{abstract}

\section{INTRODUCTION}

The processes of air-water interaction are in general use in engineering. Air and water tend to be the most popular heat-storage media. In particular, the process of air-water cooling is widely used in water cooling at thermal electric power stations in cooling towers, in systems of in-plant water recirculation, etc.

The study of heat-and-mass transfer in humid air has both theoretical and practical significance and up to the present moment it is not fully investigated, as the conventional method of mass transfer calculation on the basis of Reynolds analogy as applied to the processes connected with heat-tohumidity air conditions change turns out to be not applicable in the most cases [1].

The objective of the present study is to analyze the air-water cooling process based on experimentation.

\section{Problem Statement AND ReSearch methods.}

The given study was conducted in a bubble column of a bubble-agitated liquid cooler. The experimental installation scheme is shown in Fig. 1. 


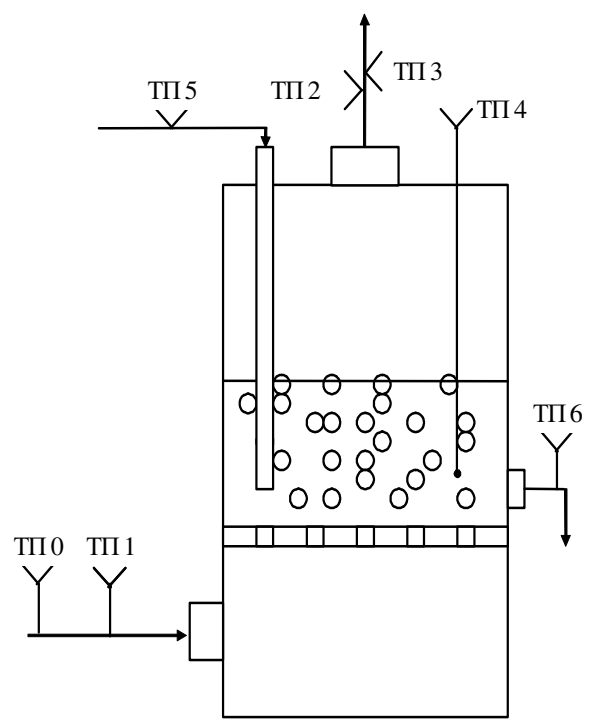

Figure 1. The scheme of experimental installation

At the inlet of the bubble-agitated liquid cooler the air with environmental parameters was delivered. Passing through the bubble column the air went into the separator, and then to the suction pipe of the blower installation. The liquid (water) was delivered out of water-supply system into the heater, then to the bubble-agitated liquid cooler grill.

In experiment cooler restriction, liquid flow, input/output gas and liquid temperature and bubble column temperature were measured.

The restriction was measured by means of U-differential meter. There was also gravimeter measurement. Gas and liquid temperatures were determined by copper-constantan thermocouples. There were estimations of gas flow necessary for calculations.

The fig. 2 shows the dependence of $\Delta T_{g}=T_{2}-T_{1}$ on $\Delta T_{l}=T_{l 1}-T_{l 2}$ where $\mathrm{T}_{1}, \mathrm{~T}_{2}, \mathrm{~T}_{11}, \mathrm{~T}_{12}$ are input/output gas and liquid temperatures correspondingly.

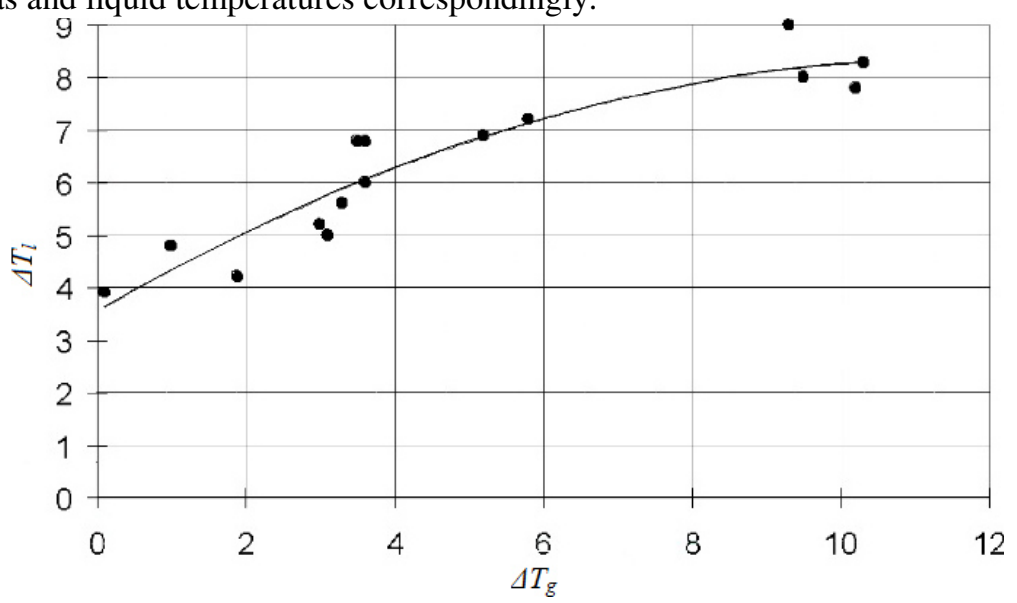

Figure 2. The dependence of differences in input/output gas temperature $\left(\Delta T_{g}\right)$ and liquid temperature $\left(\Delta T_{l}\right)$ in the bubble column

The results obtained allow us to assess the depth of cooling range reached within the experiment.

The experimental data were treated in accordance with the methods described in [1]. The equation of heat balance is the following one: 


$$
Q_{h}=Q_{i} \pm Q_{k}
$$

where $Q_{h}=\rho_{b} G_{b}\left(H_{l}-H_{2}\right)$ is full heat flux determined by changes in humid air enthalpy up to $\left(\mathrm{H}_{1}\right)$ and after $\left(\mathrm{H}_{2}\right)$ of the cooler, $\mathrm{J} / \mathrm{kg} ; \rho_{b}$ is air density in $\mathrm{kg} / \mathrm{m}^{3} ; G_{b}$ is air flow, $\mathrm{m}^{3} / \mathrm{s} ; Q_{i}=r G_{n}$ is an evaporation constituent of heat balance, W; $r$ - heat of vaporization, $\mathrm{J} / \mathrm{kg} ; Q_{k}=c_{p} G_{b}\left(t_{2}-t_{1}\right)$ - convection component of full heat flux, $\mathrm{W} ; \mathrm{t}_{1}$ and $\mathrm{t}_{2}$ are air temperatures before and after the cooler determined by dry-bulb thermometer reading; $c_{p}$ - heat capacitance, $\mathrm{J} /(\mathrm{kg} \cdot \mathrm{K})$.

Having divided left and right parts of equation (1) into $\rho c_{p} V_{0} F \Delta t$, we receive

$$
S t_{h}=S t_{d} \frac{r \overline{\Delta C}}{c_{p} \overline{\Delta t}} \pm S t_{k}
$$

The equation (2) will be written as

$$
\frac{\left|S t_{h}-S t_{k}\right|}{S t_{d}}=\frac{r \overline{\Delta C}}{c_{p} \overline{\Delta t}}
$$

Let us introduce the following notations

$$
K_{1}=\frac{\left|S t_{h}-S t_{k}\right|}{S t_{d}} ; \quad K_{2}=\frac{r \overline{\Delta,}}{c_{p} \overline{\Delta t}} .
$$

In fig. 3 there are the experimental data dependence of coefficients $\mathrm{K} 2$ and $\mathrm{K} 1$ and calculation on (3) (diagonal line).

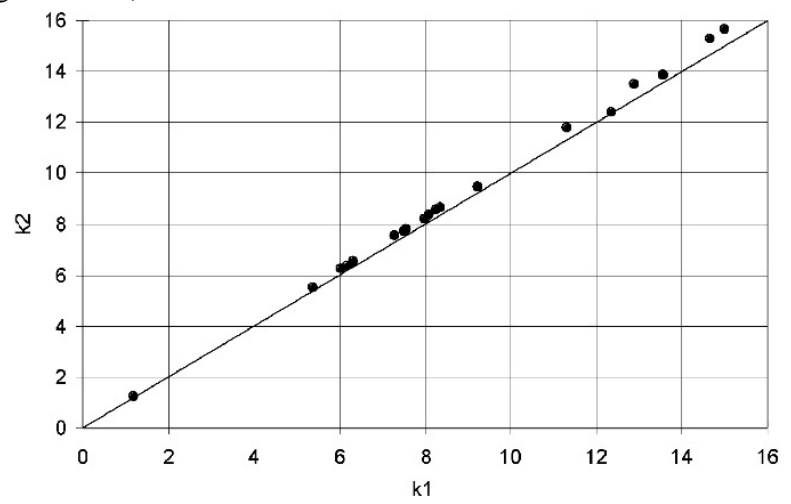

Figure 3. The dependence between the coefficients $\mathrm{K}_{1}, \mathrm{~K}_{2}$ on experience-based data and calculations With respect to deviation from the diagonal line we may predetermine accuracy in heat balance experiments

The experimental data also allow us to estimate the coefficient of efficiency of air-water cooler using a formula

$$
\eta=\frac{Q_{J b}}{Q_{U l}}
$$

where $Q_{\partial}=Q_{l}=c_{p} G\left(t_{l 1}-t_{l 2}\right)$ is heat energy given back by liquid in actual process, $Q_{u}=c_{p} G\left(t_{l 1}-t_{g m 2}\right)$ - heat energy given back by liquid in ideal process, where $t_{\mathrm{gm} 2}$ is a temperature of wet-bulb thermometer at the cooler outlet.

The fig. 4 shows the cooler coefficient of efficiency dependence on initial liquid temperature. As seen, the bulb column has enough high coefficients of process efficiency $(0,8-0,98 \%)$. 


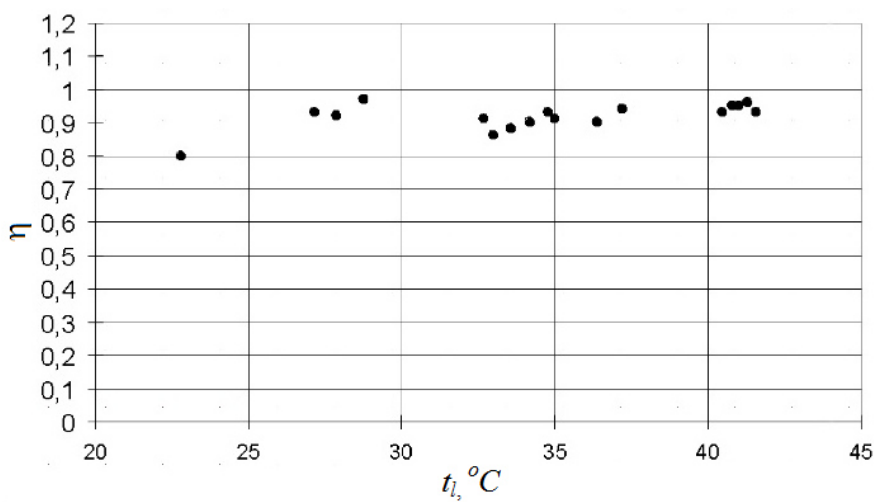

Figure 4. The dependence of $\eta$ of the cooler on initial liquid temperature

As noted above while analyzing data on mass-transfer in bubble columns the surface area of bubbling grill is used as a calculation. In such a case a mass-transfer coefficient is called "surface coefficient". The second method of determination of calculated mass-transfer coefficient is connected with estimation of complete surface of phase contact. Thus, the mass-transfer coefficient is called "true coefficient".

However, according to the present views both methods of mass-transfer coefficient estimation do not give a real indication of mass-transfer processes in bubble columns. Thus, in research of aerosol entrapment in bubble column there is one essential mechanism of particles emission out of the flow called "shock mechanism" when the basic particles emission takes place together with the shock of flow of exhaust gas on liquid surface [3]. We may assume that the presented method is more important for molecular systems where the component separation is carried out with molecular and convective diffusion methods.

In this paper when analyzing experience-based data on mass-transfer coefficient we set up the problem to determine the surface area of bubbles generating simultaneously on all the holes of the cooler grill. The computation of that surface is performed by the formula

$$
\left.F_{\sum n}=F_{p} S \frac{4}{\pi d_{0}^{2}}\right\rangle F_{n}\langle,
$$

where $F_{p}$ is grill surface, $\mathrm{m}^{2} ; S$ - relative area of grill holes, $d_{0}$ is a diameter of grill holes,

$$
\rangle F_{n}\langle=4 \pi\rangle r\left\langle^{2}\right.
$$

an average surface of one bubble in duration of rise determined by its mid-radius.

To determine the bubble mid-radius we use the equation which is obtained from the criteria of mass balance of inward gas

$$
\rho V_{0} \frac{\pi d_{0}^{2}}{4} d t=2 \pi r^{2} \rho d r
$$

After differentiation of (8) we receive the bubble departure diameter

$$
r=\left(\frac{3}{8} \frac{V_{0} d_{0}^{2}}{f}\right)^{\frac{1}{3}}
$$

where $f$ is departure frequency which can be equal to $f=20 \mathrm{~s}^{-1}$.

Using (9) it is estimated the bubble mid-radius from the starting point of its growth to its detachment

$$
\gamma r=\frac{3}{4}\left(\frac{3}{8} \frac{V_{0} d_{0}^{2}}{f}\right)^{\frac{1}{3}} .
$$

Inserting the obtained expression into the formulas (6), (7) we receive

$$
\rangle F_{n}\left\langle=2 \frac{1}{4} \pi\left(\frac{3}{8} \frac{V_{0} d_{0}^{2}}{f}\right)^{\frac{2}{3}}\right.
$$

Definitely for complete bubble surface we receive 


$$
F_{\sum n}=4.86 F_{p} S V_{0}^{\frac{2}{3}} d_{0}^{-\frac{2}{3}} f^{-\frac{2}{3}}
$$

The fig. 5 shows the mass-transfer coefficients data in calculation of which we use the area evaluated by the formula (12).

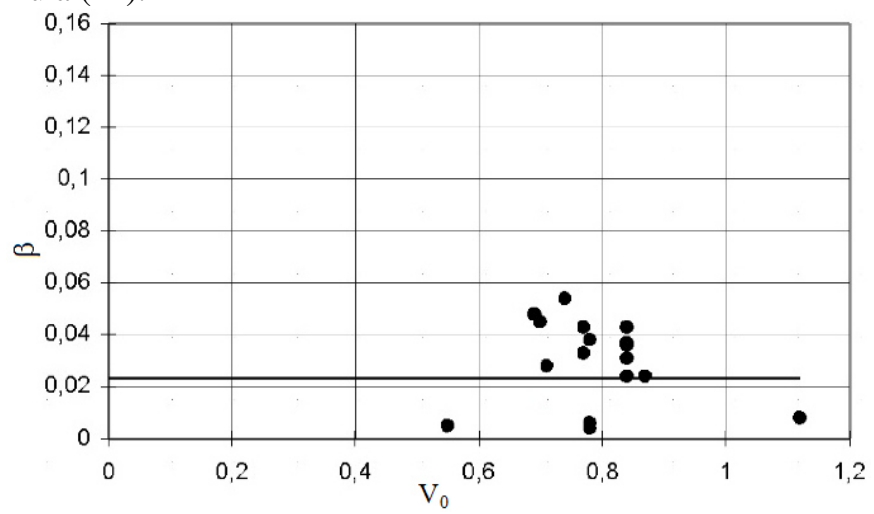

Figure 5. The dependence of mass-transfer coefficient on gas velocity

When analyzing data on mass transfer in liquid-gas systems in [4] it is defined to use the dependence for calculating true mass-transfer coefficient obtained for the first time by Higbie [5], which is also known as approximation in a short period of time of phase contact

$$
\beta=\frac{2}{\sqrt{\pi}} \sqrt{\frac{D}{\tau}},
$$

where $D$ is a diffusion coefficient, $\mathrm{m}^{2} / \mathrm{s}, \tau$ is phase contact time.

For the bubble column phase contact time is the time of bubble growth and floating-up. Let us determine the phase contact time in (13) taking into account only the first process stage as the time of the bubble growth on the grill surface up to the moment of its detachment. Then it is possible to find $\tau$ by departure frequency $f$ because $\tau=1 / f$. For the bubble column it is common knowledge that $f=20 \mathrm{~Hz}$, and with $\mathrm{D}$ equal to $2,1 \cdot 10^{-5}$ from (13) we obtain

$$
\beta=\frac{2}{\sqrt{\pi}} \sqrt{2.1 \cdot 10^{-5} \cdot 20} \approx 3.5 \cdot 10^{-2} \mathrm{~m} / \mathrm{s} .
$$

Despite the considerable scatter of experience-based data what is objectively due to the experiment conditions as it corresponds to short periods of Gukhman number $\left(\mathrm{Gu}<4 \cdot 10^{-2}\right)$ when its impact on the process of humid air-water interaction tends to be very important according to the research [6], thus we may state reasonable correlation of obtained results with experimental data.

This fact substantiates the assumption that it is stages of gas outlet from the cooler grill into the bubble that influence the mass transfer process.

\section{Conclusion}

The results of experimentation of air-water cooling in the bubble column are given. It is shown that water is cooled down almost to wet-bulb thermometer temperature with the process coefficient of efficiency equal to $0,8-0,98 \%$.

The data on mass-transfer coefficient, which while their reference to the bubble surface in wall layer are in agreement with the results of Higbie's short period phase contact, are provided. 


\section{References}

1. Burdukov A.P., Dorokhov A.R., Kazakov V.I. Massoobmen v gazovoj faze tsentrobezhnobarbotazhnogo sloya (Mass transfer in gas phase of centrifugal bubble unit) // Teplofizika i aeromekhanika. - 1996. - Vol.3, №2. - 173-179 pp.

2. Bogoslovski V.N., Kokorin O.Ya., Petrov L.V. Konditsionirovanie vozdukha b kholodosnabzhenie (Air conditioning and refrigeration): College textbook. Ed. by V.N. Bogoslovski. - M.: Strojizdat, 1985. - 367 p.

3. Shilayev M.I., Dorokhov A.R., Nechaev P.G. O mekhanizme ulavlivaniya pyli v pennykh apparatakh (On dust arresting in bubble columns) // Izvestiya vuzov. Stroitel'stvo. - 1997. №4. - 108-115 pp.

4. Akselrod Y.V. Gazozhidkostnye khemosorbtsionnye protsessy. Kinetika i modelirovanie (Gas-water chemisorption processes. Kinetics and modelling). - M.: Khimiya, 1989. - 240 p.

5. Higbie R. The rate of absorption of the pure gas in to a still liquid during short periods of exposure // Trans. Am. Chem. Eng. - 1935. - V.31. №2. - 365-389 pp.

6. Shilyaev M.I., Dorokhov A.R. Teplomassoobmen v tsentrobezhno-barbotazhnom sloye (Heat and mass transfer in centrifugal bubble units) // Izvestiya vuzov. Stroitel'stvo. - 1988. - №1. - 60-66 pp. 